\title{
Percepção de responsáveis e recreadores sobre diferentes representações gráficas de guia alimentar para crianças de dois a três anos
}

\author{
Perceptions of parents, guardians and recreators on different graphic representations of food guides \\ for young children aged two to tree years
}

\section{Roseane Moreira S. Barbosa ${ }^{1}$, Luciléia Granhen T. Colares², Eliane de Abreu Soares ${ }^{3}$}

\section{RESUMO}

Objetivo: Conhecer a percepção de responsáveis e recreadores sobre diferentes representações gráficas de guia alimentar para crianças de dois a três anos de idade.

Métodos: Estudo qualitativo feito com seis recreadores e 12 responsáveis por crianças de dois a três anos de uma creche pública do Rio de Janeiro. Um questionário sociodemográfico foi aplicado e uma entrevista semi-estruturada, realizada. Mostraram-se cinco representações gráficas (roda, pirâmide, arco-íris, trenzinho e prato) para que os participantes respondessem a perguntas sobre conhecimento dos grupos alimentares, conceito de nutrição (proporcionalidade/ moderação e variedade), alimentação saudável e escolhessem a figura que melhor representasse a alimentação saudável. Procedeu-se, então, à análise de conteúdo.

Resultados: A maioria dos responsáveis (92\%) percebeu o conceito de grupos alimentares com clareza, pois reportou que os guias estavam divididos por alimentos e estes agrupados de acordo com os nutrientes. Os participantes compreenderam o conceito de proporcionalidade/moderação, porém o formato hierarquizado da pirâmide causou dificuldade de interpretação, já que apontaram os grupos no topo como os mais importantes. Os participantes salientaram a importância de incluir na dieta alimentos de todos os grupos, porém o conceito de variedade intragrupo foi compreendido por apenas dois participantes. Os entrevistados elegeram a roda como o ícone que melhor representava a alimentação saudável.

Instituição: Universidade Federal do Rio de Janeiro (UFRJ), Rio de Janeiro, RJ, Brasil

${ }^{1}$ Nutricionista da Fundação Ataulpho de Paiva, mestre em Nutrição Humana pela UFRJ, doutoranda do curso de Nutrição Humana da UFRJ, Rio de Janeiro, RJ, Brasil

${ }^{2}$ Nutricionista, doutora Ciências na área de Saúde Pública pela Escola Nacional de Saúde Pública da Fundação Oswaldo Cruz, professor adjunto do Instituto de Nutrição Josué de Castro (INJC) da UFRJ, Rio de Janeiro, RJ, Brasil

${ }^{3}$ Nutricionista, doutora em Ciência dos Alimentos pela Universidade de São Paulo (USP), professora adjunta do Instituto de Nutrição da Universidade do Estado do Rio de Janeiro (Uerj), professora-associada do INJC da UFRJ, Rio de Janeiro, RJ, Brasil
Conclusões: Na elaboração de um guia alimentar, devese levar em consideração o entendimento dos conceitos nutricionais por parte dos indivíduos para que os objetivos propostos possam ser atingidos.

Palavras-chave: criança; nutrição; ciências da nutrição infantil.

\section{ABSTRACT}

Objective: To study the perception of parents, guardians and recreators on different graphic representations of food guides for young children aged two to three years.

Methods: Qualitative research with the participation of six recreators and 12 parents/guardians of young children aged two to three years in a public day care center of Rio de Janeiro, Brazil. Five graphic representations of nutritional concepts (circle, pyramid, rainbow, little train and plate) were shown to these adults. Then, they were questioned about food group knowledge, concepts of nutrition (proportionality, moderation and variety), healthy eating habits. After this step, they were supposed to point out the best icon to represent healthy eating habits. Analysis of the content was performed.

Results: Most of the parents/guardians (92\%) understood well the concept of food groups as they reported that food guides were divided by foods and grouped according to nutrients. They understood the concept of proportionality/

Endereço para correspondência:

Roseane Moreira S. Barbosa

Rua Visconde de Pirajá, 630/507 - Ipanema

CEP 22410-002 - Rio de Janeiro/RJ

E-mail: roseanesampaio@ig.com.br

Recebido em: 16/4/2008

Aprovado em: 14/7/2008 
moderation; however, the hierarchic format of the pyramid led them to misinterpretation: they pointed the food groups at the top as the most important ones. Participants remarked the importance of including all food groups in the diet, but the concept of intragroup variety was only understood by two participants. Adults chose the circle as the best graphic representation for a healthy nutrition.

Conclusions: The elaboration of a food guide should take into consideration the comprehension of nutritional concepts by individuals in order to achieve the goals proposed.

Key-words: child; nutrition; child nutritional sciences.

\section{Introdução}

Os hábitos alimentares e as necessidades nutricionais do homem contemporâneo começaram a ser estabelecidos na pré-história. As práticas alimentares muitas vezes sofreram adaptações para hábitos pouco saudáveis, o que constitui desvantagem para a saúde, tendo como conseqüência desvios ponderais e desenvolvimento de deficiências nutricionais múltiplas e específicas ${ }^{(1)}$.

Em 1992, foi realizada a Conferência Internacional de Nutrição em Roma, onde foram discutidas estratégias e ações para a melhoria do bem-estar nutricional e do consumo alimentar da população. De acordo com esse evento, os guias alimentares deveriam ser divulgados para diferentes grupos etários. Para atingir tal objetivo, cada país seria responsável pelo planejamento de ações de acordo com sua cultura a fim de se solucionarem os problemas de saúde relacionados a dietas, com a adaptação de estratégias locais ${ }^{(2)}$.

Os guias alimentares são desenvolvidos para ajudar os profissionais de Saúde Pública, educadores e a indústria de alimentos a orientar a população sobre alimentação saudável e, assim, prevenir excessos e carências nutricionais, uma vez que os aspectos principais da mensagem se baseiam na variedade, moderação e proporcionalidade dos alimentos que devem compor uma dieta balanceada ${ }^{(3,4)}$.

A representação gráfica dos guias alimentares tem o propósito de ajudar o grupo populacional eleito a selecionar os alimentos que deveriam ser incluídos na dieta e em que proporções, sendo que cada país deve escolher um símbolo conhecido e familiar, que possa ser facilmente utilizado pelos consumidores, pela indústria de alimentos e por profissionais de educação nutricional ${ }^{(5,6)}$. Apesar das diversas formas de representação do guia alimentar, o objetivo é o mesmo: orientar os consumidores tanto na escolha dos grupos de alimentos quanto nas porções diárias necessárias para que uma dieta seja saudável.

De acordo com Painter $e t a l^{(4)}$, os guias alimentares de diferentes países vêm sendo desenvolvidos e revisados para que estejam cada vez mais adaptados à cultura, à disponibilidade de alimentos, às preferências alimentares e ao padrão dietético da população. O desafio é encontrar a melhor forma de comunicação para promover uma alimentação saudável. Alguns países, como os Estados Unidos, México, Panamá e Chile, optaram por expressar graficamente seus guias alimentares a partir da pirâmide. O Canadá escolheu a forma de um arcoíris, a Inglaterra o formato do prato; Portugal optou pela roda, Costa-Rica pelo formato de pizza e a Guatemala optou pela representação gráfica de um pote de cerâmica. Outros países como Cuba e Venezuela não utilizam representação gráfica, apenas mensagens de fácil compreensão ${ }^{(7)}$.

No Brasil, apenas dois guias alimentares infantis foram desenvolvidos. Em 2002, a partir de uma iniciativa conjunta da Organização Pan-Americana de Saúde (OPAS) com o Ministério da Saúde foi desenvolvido o guia alimentar para crianças de seis a 23 meses, propondo-se, além da pirâmide alimentar como representação gráfica, os dez passos para atingir uma alimentação saudável ${ }^{(8)}$. Um outro guia brasileiro para crianças de dois a três anos de idade foi desenvolvido por Philippi et al $(2003)^{(9)}$, tornando-se um instrumento de orientação nutricional embasado na proposta norte-americana. Esses dois guias brasileiros infantis utilizam a representação gráfica da pirâmide alimentar, ícone proposto pelo Departamento de Agricultura dos Estados Unidos. Nos Estados Unidos, foram realizados vários estudos com representantes da indústria, profissionais da saúde e indivíduos da população para a escolha dessa representação gráfica. Entretanto, no Brasil, a pirâmide alimentar vem sendo utilizada como ferramenta de educação nutricional sem nenhum estudo prévio que avalie se ela é entendida pela população como um ícone representativo da alimentação saudável.

Algumas pesquisas mostraram que os consumidores são capazes de expressar preferências por determinadas representações gráficas ${ }^{(10-12)}$. Porém, são poucos os estudos que apontam as preferências dos indivíduos com relação aos diversos ícones alimentares. Temporini ${ }^{(13)}$ destacou a necessidade de conhecimento das maneiras de agir, sentir e pensar da comunidade para que sejam propostas medidas ou ações de Saúde Pública. Portanto, o objetivo desse estudo foi conhecer a percepção de responsáveis e recreadores sobre as diferentes representações gráficas de guias alimentares para crianças de dois a três anos de idade. 


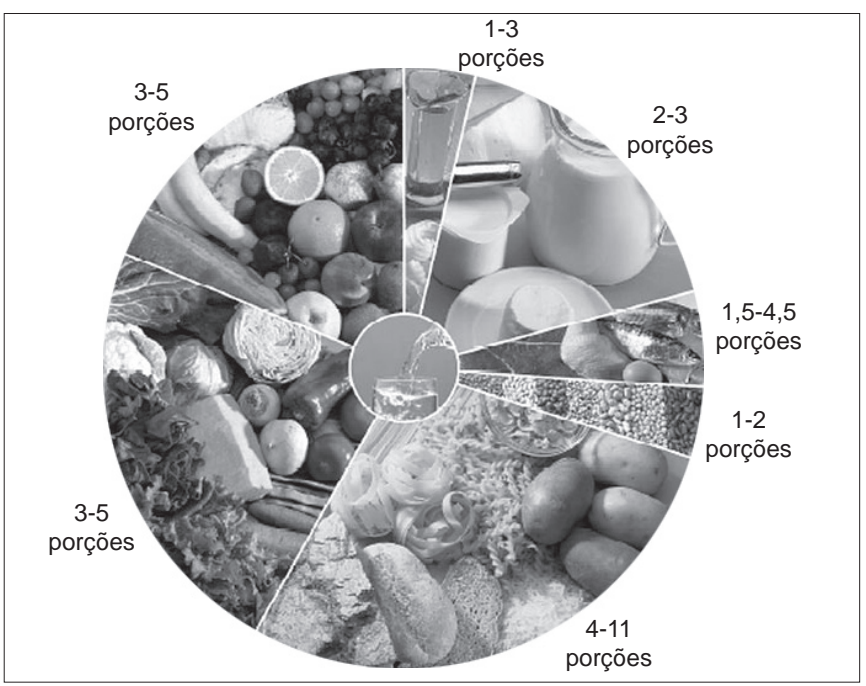

Figura 1 - Roda dos Alimentos (Fonte: Rodrigues et al(21)).

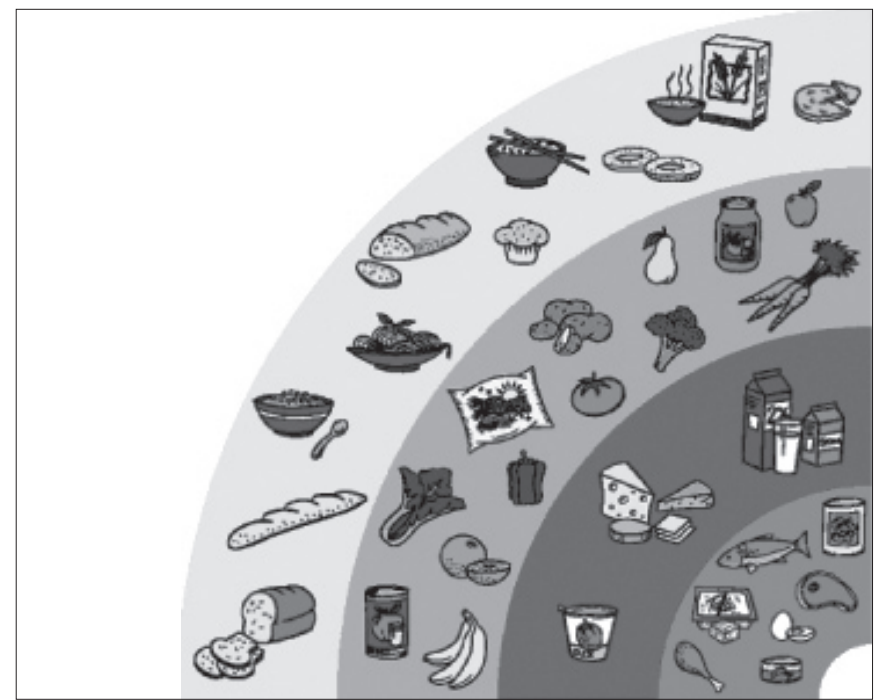

Figura 2 - Arco-íris (Fonte: ONPP(22)

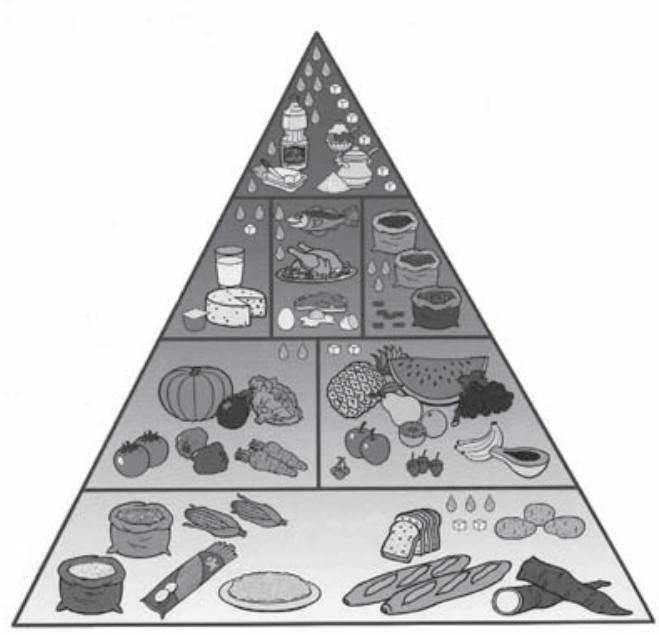

Figura 3 - Pirâmide Alimentar (Fonte: Davis ${ }^{(23)}$ ).

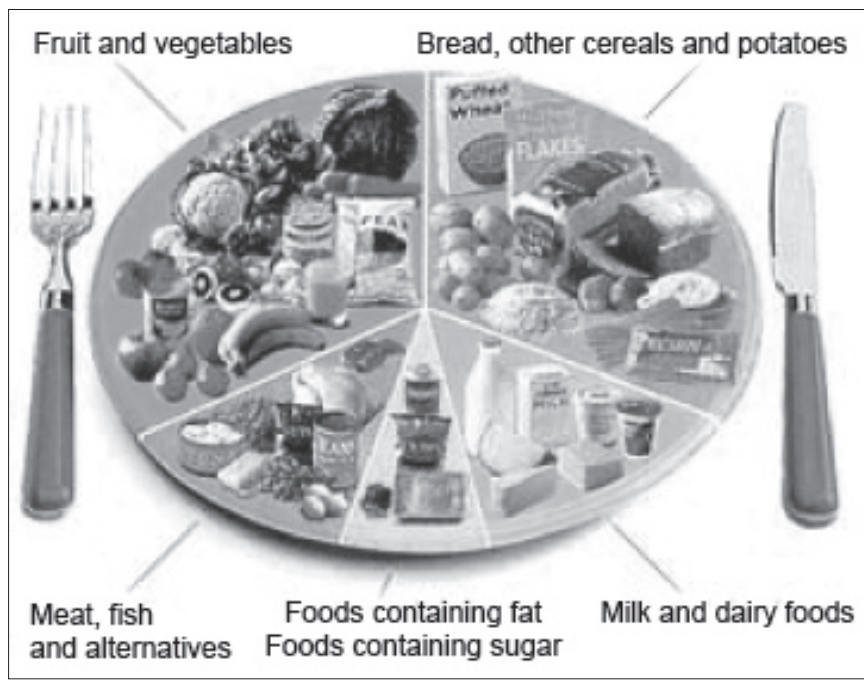

Figura 4 - Prato (Fonte: Hunt, Rayner e Gatemby ${ }^{(24)}$ ).

\section{Métodos}

O presente estudo foi desenvolvido dentro de um referencial de pesquisa qualitativa, entendida como um conjunto de práticas interpretativas que busca investigar os sentidos que os indivíduos atribuem aos fenômenos e ao conjunto de relações em que eles se inserem ${ }^{(14)}$.

Participaram da pesquisa, selecionados por conveniência ${ }^{(15)}$, seis recreadores e 12 responsáveis por crianças de dois a três anos de idade de uma creche no município do Rio de Janeiro (Brasil). Os participantes da pesquisa não receberam nenhuma orientação prévia sobre alimentação saudável. Esses dois grupos foram escolhidos por terem maior contato com as crianças e estarem envolvidos na realidade social e cultural de cada uma delas.

A coleta de dados constou de entrevista semi-estrutrada realizada na creche em um único encontro, com duração média de uma hora. Inicialmente, cada participante respondeu a um questionário sociodemográfico que trazia questões ligadas à escolaridade, à ocupação e à idade. Após o preenchimento desse questionário, cinco representações gráficas foram mostradas aos participantes: a roda dos alimentos, o prato, o arco-íris, a pirâmide e o trenzinho (Figuras 1 a 5) para que, a partir desses ícones, eles pudessem responder às questões do estudo. Tais ícones alimentares foram selecionados pelo fato de serem utilizados como guias alimentares para crianças em outros países; o trenzinho, porém, foi elaborado pelos pesquisadores deste estudo para apresentar os conceitos nutricionais de forma lúdica e dialógica com o universo infantil. O trenzinho é composto por oito vagões, cada um deles representa um grupo alimentar: cereais, hortaliças, frutas, leguminosas, leite, carnes, açúcares e gorduras. 


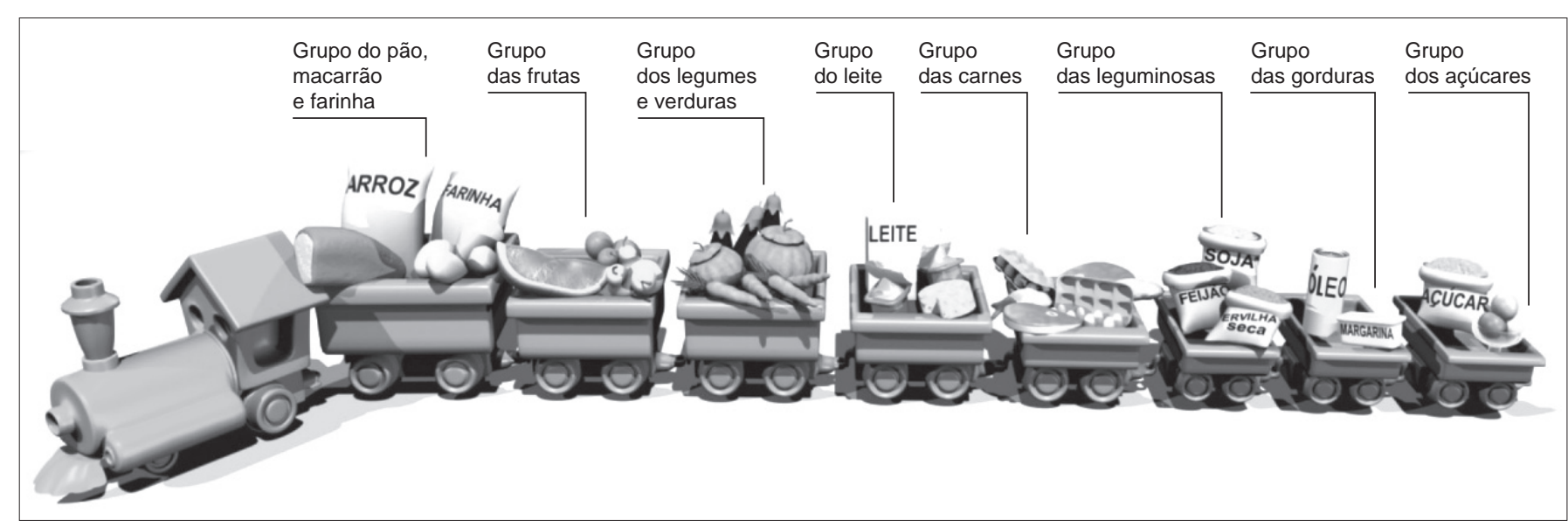

Figura 5 - Trenzinho (Elaborado pelo grupo de pesquisa da UFRJ)

Durante a entrevista, procurou-se estabelecer uma conversa dirigida com os entrevistados em torno dos temas que integravam o objeto do estudo, ou seja, questões relativas ao conhecimento dos grupos alimentares, aos conceitos da nutrição (proporcionalidade e variedade) e à alimentação saudável. Essas entrevistas tiveram certa flexibilidade tanto na ordem das questões quanto na forma de abordagem, adequando-se sempre ao entrevistado. Por fim, foi solicitada a escolha da figura que melhor representasse a alimentação saudável. Todas as entrevistas foram gravadas e transcritas pelo mesmo pesquisador.

Após a transcrição das entrevistas, analisou-se o conteúdo ${ }^{(16)}$. Essa etapa envolveu leitura flutuante e, posteriormente, leitura exaustiva para indentificar os temas mais importantes presentes nas falas dos participantes. Durante o procedimento, as informações foram agrupadas e categorizadas para que fosse possível estudar a percepção de responsáveis e recreadores acerca de diferentes representações gráficas de guia alimentar para crianças com idade entre dois e três anos.

Esta pesquisa foi aprovada pelo Comitê de Ética em Pesquisa do Instituto de Puericultura e Pediatria Martagão Gesteira da Universidade Federal do Rio de Janeiro e pela Secretaria Municipal de Educação. Todos os participantes foram informados sobre os objetivos do estudo e os procedimentos utilizados e assinaram o termo de consentimento livre e esclarecido.

\section{Resultados}

A partir do questionário sociodemográfico, observou-se que cinco (83\%) recreadores possuíam ensino médio completo, apenas um concluíra o ensino superior e quatro (67\%) tinham entre 21 e 30 anos. Dentre os responsáveis, verificouse que dez (88\%) possuíam ensino fundamental incompleto, sete $(58 \%)$ encontravam-se na faixa etária dos 31 aos 40 anos e oito $(67 \%)$ tinham como ocupação o serviço doméstico.

Os relatos mostram o conhecimento dos responsáveis de que os guias alimentares estão divididos por alimentos e que estes são agrupados de acordo com os nutrientes. Em relação à proporcionalidade/moderação, observou-se que os participantes compreenderam que os grupos alimentares possuíam tamanhos diferentes. A roda foi o ícone alimentar mais escolhido, seguido do trem. A análise de conteúdo do discurso dos entrevistados, que nos levou a esses resultados, será analisada junto à discussão.

\section{Discussão}

De acordo com a Food and Nutrition Board da Organização Mundial de Saúde (OMS) ${ }^{(17)}$, a principal razão para o desenvolvimento dos guias dietéticos com base nos alimentos é o fato de as informações baseadas nos alimentos serem mais facilmente compreendidas pelos consumidores do que aquelas baseadas nos nutrientes. Neste estudo procurou-se perceber como os responsáveis e educadores compreendiam os conceitos da nutrição por meio de diversas representações gráficas.

Os grupos alimentares são constituídos de alimentos com os mesmos nutrientes. Saber identificá-los permite uma melhor compreensão acerca das substituições adequadas dos alimentos nas refeições. Dessa maneira, procurou-se avaliar se os participantes entendiam a presença dos alimentos em diferentes grupos alimentares. As falas dos recreadores e responsáveis foram: "valor nutricional", "há grupos que se pode comer à vontade" e "quantidades necessárias" (recreadores), “... porque alguns alimentos têm mais e outros têm menos 
vitaminas", "Está separado em carne, leite, frutas, verduras, legumes", "Divide-se em frutas, verduras, leite", "De acordo com o valor calórico e nutritivo", "Está dividido em feijão, que contém mais ferro, alimentos mais gordurosos, e frutas e verduras que têm vitaminas"(responsáveis).

Os relatos mostram a crença dos responsáveis na divisão por alimentos dos guias alimentares, bem como seu agrupamento de acordo com os nutrientes. O conceito nutricional de grupo alimentar foi entendido pela maioria dos responsáveis (92\%), o que não foi observado nas falas dos recreadores por não serem muito claras. Apenas um responsável apresentou dificuldade no entendimento dos grupos alimentares, incluindo o feijão no grupo dos cereais, com a seguinte fala: "No grupo do leite tem iogurte e queijo, no grupo das carnes tem o peixe, o outro tem pão, macarrão, feijão, arroz". Essa fala refletiu a dificuldade de identificação do grupo das leguminosas.

Achados similares foram encontrados na pesquisa de Volster $e t a^{(18)}$. Os autores avaliaram a compreensão do guia sul-africano por meio de grupos focais, com mulheres da área urbana e rural. Os resultados mostraram que as participantes não compreenderam algumas mensagens com relação a determinadas terminologias e conceitos, tais como "leguminosas", "alimentos de origem animal" e "lanches mais saudáveis”"(18).

Alguns conceitos básicos de nutrição referem-se à proporcionalidade/moderação e à variedade. De acordo com o Centro para Promoção de Nutrição ${ }^{(6)}$, o guia alimentar deve divulgar esses conceitos nutricionais à população por meio da representação gráfica.

Em relação à proporcionalidade/moderação, observou-se que os participantes compreenderam que os grupos alimentares possuíam tamanhos diferentes, sendo que a ingestão dos alimentos dos grupos com maior número de porções deveria ser feita em maior quantidade: "Tem que ser em quantidade moderada, nada em excesso" (recreadores); "A divisão é de acordo com os nutrientes que o alimento tem como, por exemplo, as gorduras", "A necessidade do dia a dia, devido à importância, devido à quantidade” (responsáveis).

Apesar de ter havido identificação do entendimento dos participantes sobre a proporcionalidade/moderação dos grupos alimentares nas diversas figuras, a pirâmide não teve a mesma compreensão por um dos recreadores, que relatou: "Na pirâmide, a dificuldade é maior. Eu ia começar pela ponta da pirâmide". Essa fala atribuiu maior importância aos grupos dos açúcares e das gorduras, não pelo seu tamanho, mas por estarem no topo da pirâmide, o que poderia evidenciar uma hierarquia dos alimentos.
Achados semelhantes foram encontrados no estudo de Achterberg ${ }^{(19)}$ que avaliou o entendimento do guia alimentar americano e mostrou que a pirâmide não foi compreendida pelos consumidores. Houve dificuldade de entendimento porque os grupos de alimentos que se encontravam no topo ou na base da pirâmide eram considerados mais ou menos importantes pelos consumidores. Além disso, os consumidores entenderam que as gotas brancas de gorduras e açúcares espalhadas nos grupos alimentares eram defeitos de impressão, além de não compreenderem o conceito de porção.

Resultados similares foram demonstrados por Domper $e t$ $a l^{(20)}$. Os autores avaliaram um programa de educação nutricional para consumidores de um supermercado. Os consumidores ficaram confusos com a pirâmide, pois indicava maior consumo dos alimentos apresentados na base e, na visão dos participantes, tais alimentos forneciam excesso de energia. Portanto, a hierarquização da pirâmide pode confundir os consumidores nas escolhas adequadas dos grupos de alimentos para uma alimentação saudável. Nesse contexto, a roda e o prato ainda são utilizados como guias alimentares especificamente em Portugal e na Inglaterra. Eles têm o formato de um círculo dividido em grupos de alimentos de diferentes tamanhos e, ao contrário da pirâmide, não hierarquizam os alimentos, mas atribuem a mesma importância a todos eles ${ }^{(22)}$.

Outro relato mostrou que um responsável não conseguiu entender que todos os grupos alimentares são importantes, referindo o entendimento incorreto sobre os benefícios das gorduras na alimentação: "O organismo precisa de tudo um pouco, sendo que as frutas e legumes estão no mesmo patamar que o arroz e feijão, já a gordura, não". Apesar do relato acima, os outros participantes salientaram que todos os grupos alimentares são importantes: "Todos são importantes, mas em quantidade moderada"; "Todos são importantes, mas a quantidade é diferente" (recreadores); "A gente precisa de tudo um pouco, os legumes e as frutas o organismo absorve melhor" (responsáveis).

Para realizar uma alimentação balanceada é necessário incluir todos os grupos alimentares, além de ser indispensável a variedade dos alimentos de um mesmo grupo. Com relação à compreensão de uma alimentação saudável, os relatos foram: "Uma alimentação balanceada com carne, legumes, verduras, pães, feijão, grãos, derivados de leite", "É comer um pouco de verdura, legume, feijão, comer um pouco de cada alimento", "Dieta bem montada onde tenha legumes, verduras e grãos" (recreadores), "Alimentação com frutas, legumes, ovos, arroz, feijão, leite e peixe", "Alimentação com frutas, legumes, ovos, arroz, feijão, leite até certa idade e peixe", "Muitos legumes, verduras, menos carne vermelha", "Não consumir alimentos enlatados, tomar leite, comer arroz e feijão, frutas” (responsáveis). 
Tanto os recreadores quanto os responsáveis mostraram um conhecimento adequado sobre os grupos alimentares que devem ser incluídos nas refeições diariamente. Destaca-se que, em todas as falas, os dois primeiros alimentos citados são as frutas e os legumes, demonstrando a associação desses grupos com uma alimentação saudável. Porém, o conceito de variedade intragrupo (variedade dos alimentos de um mesmo grupo) não foi relatado pela maioria dos participantes, havendo menção somente à variedade dos grupos alimentares. Apesar de não ter sido completamente compreendido o conceito de variedade pelos participantes, um recreador acrescentou a fala: "Uma alimentação adequada com variedade", "Não tirar o que gosta, mas renovar sempre".

A representação gráfica do arco-íris chamou a atenção de três participantes por causa das cores. Nesses relatos, o conceito de variedade dos alimentos também foi mencionado, pois as refeições coloridas, além de agradarem a visão e aguçarem o paladar, fornecem diferentes nutrientes: "As refeições têm que ser momentos de alegria" (recreadores); "Dieta bem colorida e nutritiva para chamar a atenção das crianças", "Alimentos divididos em diferentes cores" (responsáveis). Todos os participantes concordaram que todas as figuras representavam uma alimentação saudável, sendo a roda o ícone alimentar mais escolhido, seguido do trem. Isso mostra a necessidade de se avaliar se realmente a pirâmide alimentar é o melhor ícone de representação da alimentação saudável para a nossa população.

Por se tratar de um estudo de caso, os resultados aqui apresentados não podem ser generalizados, o que constitui

\section{Referências bibliográficas}

1. Ramalho RA, Saunders $C$. The nutrition education role in the combat against micronutrient deficiencies. Rev Nutr 2000;13:11-6.

2. Food and Agriculture Organization/World Health Organization [homepage on the internet]. FAO Celebrates 50 Years - final report of the conference, Rome, December 1992. [Cited: 2006 jun 3]. Available from: http://www.fao.org/docrep/ V7700T/v7700t00.htm\#Contents

3. Ballam R. Guidelines on educational materials concerned with nutrition. Nutr Food Sci 1996;96:12-6.

4. Painter J, Rah JH, Lee YK. Comparison of international food guide pictorial representations. J Am Diet Assoc 2002;102:483-9.

5. Lanzillotti HS, Couto SR, Afonso FM. Food guide pyramids: a semiotic way of reading. Rev Nutr 2005;18:785-92.

6. Center for Nutrition Policy and Promotion. Notice of proposal for food guide graphic presentation and consumer education materials. Fed Regist 2004;69:42030-3.

7. Peña M. Guias de Alimentación en América Latina. In: $1^{\circ}$ Workshop Instituto Danone, editor. Alimentação equilibrada para a população brasileira, 1998, Florianópolis. Florianópolis: Instituto Danone; 1998. p. 31-43. uma limitação desta pesquisa. Porém, acredita-se que profissionais e pais, em muitas instituições brasileiras públicas ou privadas que cuidam de crianças dessa faixa etária, possam ter percepções semelhantes acerca do objeto de estudo.

Conclui-se que os grupos alimentares nas diversas representações gráficas dos guias dietéticos foram mais bem compreendidos pelos responsáveis do que pelos recreadores. Os participantes do estudo foram capazes de entender o conceito de proporcionalidade/moderação, mas o formato hierarquizado da pirâmide confundiu os indivíduos, por aparentemente indicar uma maior importância dos grupos alimentares que estão no topo. $\mathrm{O}$ conceito de variedade intragrupo não foi relatado pela maioria dos participantes, embora tenha havido relatos da variedade dos grupos alimentares. Os participantes compreenderam a importância da inclusão de alimentos de todos os grupos na dieta, o que sugere que eles compreendem a alimentação saudável em termos qualitativos. Em relação às representações gráficas, o ícone que melhor representou a alimentação saudável para os participantes foi a roda, seguida do trem. Isso demonstra uma necessidade de avaliação da pirâmide alimentar como o ícone de escolha para representar a alimentação saudável para a população infantil brasileira. Por fim, na elaboração de um guia alimentar deve-se levar em consideração o entendimento dos conceitos nutricionais pelo público alvo para que os objetivos propostos sejam atendidos e para que haja efetivamente uma adesão à alimentação equilibrada. Além disso, é preciso definir as estratégias de desenvolvimento, implementação e avaliação do guia, de acordo com o grupo populacional a ser atingido.
8. Ministério da Saúde. Secretaria de Política de Saúde. Organização Pan-Americana de Saúde. Ministério da Saúde. Guia alimentar para crianças menores de 2 anos - normas e manuais técnicos, n.107. Brasília: Ministério da Saúde, 2002.

9. Philippi ST, Cruz AT, Colucci AC. Pirâmide alimentar para crianças de 2 e 3 anos. Rev Nutr 2003;16:5-19.

10. No authors listed. An evaluation of dietary guidance graphic alternatives: the evolution of the eating right pyramid. Nutr Rev 1992;50:275-82.

11. Black A, Rayner M. Just read the label: understanding nutrition information in numeric, verbal and graphic formats. London: HMSO; 1992.

12. Levy AS, Fein SB, Schucker RE. More effective nutrition label formats are not necessarily preferred. J Am Diet Assoc 1992;92:1230-4.

13. Temporini ER. Pesquisa de oftalmologia em saúde pública: considerações metodológicas sobre fatores humanos. Arq Bras Oftal 1991;54:279-81.

14. Gomes R, Nascimento EF, Rebello LE, Araújo FC. As arranhaduras da masculinidade: uma discussão sobre o toque retal como medida de prevenção do câncer prostático. Ciênc Saúde Coletiva 2007;1:2-11.

15. Gil AC. Métodos e técnicas de pesquisa social. $5^{a}$ ed. São Paulo: Atlas 1999. 
16. Minayo MC. O desafio do conhecimento: pesquisa qualitativa em saúde. $2^{\text {a }}$ ed. São Paulo: Hucitec; 1993.

17. Food and Nutrition Board/World Health Organization [homepage on the internet]. Preparation and use of food based dietary guidelines. Report of joint FAO/WHO consultation, Geneva, 1996. [Cited 2006 Jun 15]. Available from: http://www.fao.org/DOCREP/x0243e/x0243e00.htm

18. Vorster HH, Love P, Browne C. Development of food-based dietary guidelines for South Africa - the process. S A J Clin Nutr 2001;14 (Suppl 3):3-6.

19. Achterberg C. Development of FBDG: collection and use of data. In: ILSI Europe, editors. National food based dietary guidelines: experiences, implications and future directions. Budapest: ILSI; 2004.

20. Domper A, Zacarias IH, Olivares SC, Hertrampf E. Evaluatión de un programa de información en nutrición al consumidor. Rev Chil Nutr 2003;30:43-51.
21. Rodrigues SS, Franchini B, Graça, P, Almeida MD. A new food guide for the Portuguese population: development and technical considerations. J Nutr Educ Behav 2006;38:189-95.

22. Office of Nutrition Police and Promotion [homepage on the Internet]. Canada's food guide to healthy eating 2002. [Cited 2008 Jul 05]. Available from: http:// www.hc-sc.gc.ca/fn-an/food-guide-aliment/index-eng.php

23. Davis CA, Escobar A, Marcoe KL, Tarone C, Shaw A, Saltos S et al. Food guide pyramid for young children 2 to 6 years old: technical report on background and development. Washington: U.S. Department of Agriculture, Center for Nutrition Policy and Promotion; 1999.

24. Hunt $P$, Rayner M, Gatenby S. A national food guide for UK: background and development. J Hum Nutr Diet 1995;8:315-22. 